\title{
Lipids Based Docosahexaenoic Acid (DHA) Carriers and their Ability to Deliver DHA to the Brain: A Prospective Outline
}

\section{Su Chen*}

Chainon Neurotrophin Biotechnology INC, San Antonio, TX 78230, USA

\section{Editorial}

The most common age-dependent neurodegenerative diseases such as dementia and Alzheimer's disease (AD) have become an urgent public health problem in the most areas of the world. Because this is a progressive brain disorder, the cure becomes much more difficult or even impossible if the prevention or treatment starts at a later stage of the diseases. For an ideal drug for the progressive disorders, however, it should enable to both simultaneously delay or halt the underlying pathological process and improve learning and memory. For example, the use of cholinesterase inhibitors such as Aricept drug has become the main and current approach to symptomatic treatment, but the agents cannot inhibit apoptosis of neurons. On the other hand, the side-effect of using chemically synthesized compounds is also another important issue.

Docosahexaenoic acid (DHA; 22:6n-3; docosa: 22 carbon atoms; hexaenoic: 6 double bonds; $n-3$ : the position of the first double bond, counting from the methyl end) is the main polyunsaturated fatty acid in the brain membrane [1]. Compared with other saturated and non-polyunsaturated fatty acids, multiple double bonds within DHA molecule can cause carbon-carbon chains to become more curved. The more kinked the fatty acid chain is, the more space it may take up, when DHA is built into neural membrane phospholipids (PL). It recently reports that the deficits in both accumulation of DHA and plasmalogen PLs in brains and circulation of AD patients [2,3] can reduce both normal levels of neural DHA PL molecular species and membrane endogenous anti-oxidation, leading to causing neuronal apoptosis and resulting in markedly influencing learning and memory.

Since the first studies on the brain health impacts of DHA from fish were published in the early 1970's, the amount of evidence suggesting a positive therapeutic role of the fatty acid has been mounting. Although a number of investigations by "in vitro incubation of highly pure DHA with various neurons in test tubes" have shown the pharmacological effects of DHA, it is still an intriguing question whether the exogenous introduction of naturally occurring DHA - containing lipids extracted from the marine source may deliver DHA into the brain in order to meet the in vitro laboratory results. Clinical studies [4] indicate that dietary DHA connecting PLs have shown better effects than DHA connecting triglycerides in improving memory and learning functions. For example, triglycerides based DHA carriers (represented by algae oil product with $40-45 \%$ DHA molecular species) showed almost no function to slow down memory and cognitive declines in people with mild to moderate AD. For other examples, the current sn-2-DHA phosphatidylcholine (PC; represented by Krill oil product with 10-15\% DHA molecular species) is the major form of PL dietary supplement; the clinical trial of Krill oil for delaying early stage AD has been completed in July 2011 [5], but there is no positive study results posted and published so far; the bovine cortex phosphatidylserine (PS), which was the first cholinergic drug in Europe to alleviate and treat $\mathrm{AD}$, has been documented. Interestingly, although both algae and Krill oils contain DHA molecular species, their therapeutic effects are different from these of the bovine cortex PS. It strongly suggests significant importance on the relationship between the chemical structure and bioavailability of various lipids based DHA carriers when they are used as the brain DHA donors in the prevention and treatment of agedependent neurodegenerative diseases. A new question is that what are the key points in the selection of the structured lipids based DHA carriers for the brain DHA donors?

On the basis of the metabolic role of PLs in vivo (the deacylation/ reacylation circle), it could be explained by the facts that the pancreatic phospholipase $\mathrm{A}_{2}\left(\mathrm{PLA}_{2}\right)$ in the intestine is largely responsible for the hydrolysis of dietary PC species, resulting in the generation of lysoPCs and free fatty acids such as DHA from the $s n-2$ position of PL molecular species. Once absorbed by the intestinal epithelium, the free DHA is esterified with either lysoPC or monoacylglycerol and transported in the chylomicrons to various tissues. Majority of the free DHA released by pancreatic PLA (PC deacylation) ends up in the triglycerides of chylomicrons. Furthermore, results from earlier studies on monkey brain and rat liver microsomal acylation systems show that the lysoPC acyltransferases exhibit lower selectivity for DHA, compared with other fatty acids (lysoPC reacylation). These results indicate that the $s n-2$ DHA of dietary PCs will not be retained in the chylomicron PLs. It was also shown that sn-2-DHA PCs were synthesized in the liver mainly from the methylation of DHA phosphatidylethanolamine (PE) rather than via the CoA-dependent esterification of lysoPC with DHA. When the PE methyltransferases were knocked out in mice, the animal was unable to maintain a normal concentration of all choline metabolites even with a supplemental source of dietary choline.

Overall, it should be pointed out here that the efficacy of dietary PLs as a source of brain DHA depends upon not only the phospholipase activities in humans (mainly human plasma), but also the location of DHA linked to the glycerol backbone of its PL carriers or the nature of the carriers to phospholipase hydrolysis (PL deacylation). A prospective outline for the new strategy is that the ideal PL carriers for supplementing brain DHA should enable to "preserve" the DHA chain in carriers in the deacylation/reacylation circle. For examples, (i) the type 1 carrier should contain DHA in the $s n-1$ position, such as $s n$-1-DHA PCs or $s n$-1-LysoPCs, which may be advantageous because the DHA would escape the hydrolysis by pancreatic PLA $\mathrm{P}_{2}$ and thus be retained in PLs during the process of transportation to the liver and

*Corresponding author: Su Chen, Chainon Neurotrophin Biotechnology INC, San Antonio, TX 78230, USA, E-mail: chens54331@yahoo.com

Received February 23, 2013; Accepted February 26, 2013; Published March 03, 2013

Citation: Chen S (2013) Lipids Based Docosahexaenoic Acid (DHA) Carriers and their Ability to Deliver DHA to the Brain: A Prospective Outline. J Bioequiv Availab 5: e28. doi:10.4172/jbb.10000e28

Copyright: (c) 2013 Chen S. This is an open-access article distributed under the terms of the Creative Commons Attribution License, which permits unrestricted use, distribution, and reproduction in any medium, provided the original author and source are credited. 
Citation: Chen S (2013) Lipids Based Docosahexaenoic Acid (DHA) Carriers and their Ability to Deliver DHA to the Brain: A Prospective Outline. J Bioequiv Availab 5: e28. doi:10.4172/jbb.10000e28

Page 2 of 2

incorporation into the lipoproteins. The $s n$-1-DHA linkage would also escape the degradation by all of other phospholipases present in plasma and liver, which also hydrolyze the $s n-2$ acyl linkage, followed by crossing the $\mathrm{BBB}$ to generate free DHA with endothelial lipase [6]; and (ii) the type 2 carrier should have the slow rates to various PLA $_{2}$ hydrolysis during the metabolism, such as DHA PS or DHA plasmanylcholine. Although it has not been accepted widely by the preliminary results that polyunsaturated PL molecular species could directly cross intestinal barriers without hydrolysis [7]; and lipoprotein particles can cross the BBB in Drosophila [8] and the lipoproteins act as transporters of therapeutic lipid molecules across the BBB for the treatment of brain disorders, the further investigations in humans do need to confirm in order to further explain the metabolic roles of the new structured PL carriers.

Finally, it should state that the current approach to supplement DHA to the brain by the structured PL carriers [9] should be the further development because at the present it is the unique possibility of having the long period of time for the administration of the agents that have limited side-effects for delaying or halting the process of agedependent neurodegenerative disorders such as dementia and $\mathrm{AD}$.

\section{References}

1. Calon F, Lim GP, Yang F, Morihara T, Teter B, et al. (2004) Docosahexaenoic acid protects from dendritic pathology in an Alzheimer's disease mouse model. Neuron 43: 633-645

2. Wood PL (2012) Lipidomics of Alzheimer's disease: current status. Alzheimers Res Ther 4: 5.

3. Goodenowe DB, Cook LL, Liu J, Lu Y, Jayasinghe DA, et al. (2007) Periphera ethanolamine plasmalogen deficiency: a logical causative factor in Alzheimer's disease and dementia. J Lipid Res 48: 2485-2498.

4. Cunnane SC, Chouinard-Watkins R, Castellano CA, Barberger-Gateau P (2013) Docosahexaenoic acid homeostasis, brain aging and Alzheimer's disease: can we reconcile the evidence? Prostaglandins Leukot Essent Fatty Acids 88: 61-70.

5. http://clinicaltrials.gov/show/NCT00867828

6. Chen S, Subbaiah PV (2007) Phospholipid and fatty acid specificity of endothelia lipase: potential role of the enzyme in the delivery of docosahexaenoic acid (DHA) to tissues. Biochim Biophys Acta 1771: 1319-1328.

7. Hossain Z, Kurihara H, Hosokawa M, Takahashi K (2006) Docosahexaenoic acid and eicosapentaenoic acid-enriched phosphatidylcholine liposomes enhance the permeability, transportation and uptake of phospholipids in Caco2 cells. Mol Cell Biochem 285: 155-163.

8. Brankatschk M, Eaton S (2010) Lipoprotein particles cross the blood-brain barrier in Drosophila. J Neurosci 30: 10441-10447.

9. http://www.benthamscience.com/coc/HT/Su-Chen.pdf 